EPJ Web of Conferences 16, 04001 (2011)

DOI: $10.1051 /$ epjconf/20111604001

(C) Owned by the authors, published by EDP Sciences, 2011

\title{
Spectroscopic detection and characterisation of planetary atmospheres
}

\author{
J.R. Barnes ${ }^{1, a}$, H.R.A. Jones ${ }^{1}$, T.S. Barman², H.R.A. Jones ${ }^{1}$, R.J. Barber ${ }^{3}$, \\ B.M.S. Hansen ${ }^{4}$, L. Prato ${ }^{2}$, E.L. Rice ${ }^{4}$, C.J. Leigh ${ }^{5}$, A. Collier Cameron ${ }^{6}$, \\ D.J. Pinfield ${ }^{1}$, J.S. Jenkins ${ }^{7}$ and D. Segransan ${ }^{8}$ \\ ${ }^{1}$ Centre for Astrophysics Research, University of Hertfordshire \\ ${ }^{2}$ Lowell Observatory, Planetary Research Centre, 1400 West Mars Hill Road, Flagstaff, \\ AZ 86001, USA \\ ${ }^{3}$ Departments of Physics and Astronomy, University College London, London WC1E 6BT, UK \\ ${ }^{4}$ Department of Physics and Astronomy, and Institute of Geophysics and Planetary Physics \\ ${ }^{5}$ University of California Los Angeles, Los Angeles, CA 90095, USA \\ ${ }^{6}$ Astrophysics Research Institute, Liverpool John Moores University, Birkenhead CH41 1LD, \\ UK \\ ${ }^{7}$ SUPA, School of Physics and Astronomy, University of St Andrews, Fife KY16 9SS, UK \\ ${ }^{8}$ Universidad de Chile, Casilla Postal 36D, Santiago, Chile \\ ${ }^{9}$ Observatoire de Genève, 51 Chemin des Maillettes, 1290 Sauverny, Switzerland
}

\begin{abstract}
Space based broadband infrared observations of close orbiting extrasolar giant planets at transit and secondary eclipse have proved a successful means of determining atmospheric spectral energy distributions and molecular composition. Here, a ground-based spectroscopic technique to detect and characterise planetary atmospheres is presented. Since the planet need not be transiting, this method enables a greater sample of systems to be studied. By modelling the planetary signature as a function of phase, high resolution spectroscopy has the potential to recover the signature of molecules in planetary atmospheres.
\end{abstract}

\section{INTRODUCTION}

The vast majority of exoplanets do not transit their host stars and have been detected via the radial velocity method. Many of these planets orbit stars with typical magnitudes of $V<9-10$. A growing number of planets are also being identified by transit surveys, which typically target fainter stars with $V>9$. However, to date, only a handful of systems have been the subject of followup characterisation studies, largely because the techniques used require that systems be both bright and transiting. Optical studies are limited to the very brightest systems, where sufficient $\mathrm{S} / \mathrm{N}$ can be attained on reasonable timescales to enable the possibility of detecting the planetary signal at high contrast. A small number of planets have been studied at near-mid infrared wavelengths where contrast ratios are more favourable and important molecules exhibit measurable broadband signatures. Of the 31 planets orbiting host stars with $K<9$, and with $M_{p} \sin (i)>0.2 \mathrm{M}_{\text {Jup }}$ and $a<0.06 \mathrm{AU}^{1}$ (i.e. highly irradiated close orbiting extrasolar giant planets), only 10 are transiting. In order to determine the atmospheric properties of a wider range of targets, a method which does not rely on transits is required.

ae-mail: j.r.barnes@herts.ac.uk

1 www. exoplanet.eu

This is an Open Access article distributed under the terms of the Creative Commons Attribution-Noncommercial License 3.0, which permits unrestricted use, distribution, and reproduction in any noncommercial medium, provided the original work is properly cited. 


\section{EPJ Web of Conferences}

Space based broadband photometry and low resolution transit spectroscopy have provided the first evidence for water and organic molecules in the atmospheres of close orbiting extrasolar giant planets (CEGPs). While a detection of $\mathrm{H}_{2} \mathrm{O}$ absorption in the optical spectra of $\mathrm{HD} 209458 \mathrm{~b}$ has been claimed [1], the first detection of $\mathrm{H}_{2} \mathrm{O}$ in the atmosphere of $\mathrm{HD}$ 189733b [2] led to the identification of $\mathrm{CO}$ and $\mathrm{CH}_{4}$ in transit spectra which probe the terminator of the planet [3]. Most recently NICMOS data have been use to infer the additional presence of $\mathrm{H}_{2} \mathrm{O}, \mathrm{CO}$ and $\mathrm{CO}_{2}$ in the dayside spectra of both $\mathrm{HD}$ 189733b [4] and HD 209458b [5].

The role of parameterised high absorbing species in irradiated CEGP atmospheres has been investigated [6] following work that suggested that TiO and VO may be depleted by the "cold trap" effect [7]. Others have nevertheless focused on the effects of TiO and VO with similar results [8], namely that the absorbing species lead to a temperature inversion and the formation of a stratosphere. For cooler atmospheres, absorbing species are expected to "rain out" and the dayside model spectra are found to be dominated by $\mathrm{H}_{2} \mathrm{O}$ and $\mathrm{CO}$ absorption lines. However, the most highly irradiated atmospheres, which possess a stratosphere, are expected to exhibit $\mathrm{H}_{2} \mathrm{O}$ and $\mathrm{CO}$ in emission. While HD 189733b and TrES-1 appear to possess atmospheres [6,9] dominated by absorption features, systems such as HD 209458b, HD 149026b and $v$ And b are under the influence of higher irradiation from their parent stars [8]. These latter systems are among those which possess atmospheres most consistent with the presence of a stratosphere $[6,10,11]$.

With the aim of observing non-transiting systems and extending space based mid-infrared measurements of planet/star contrast ratios into the near infrared, model spectra may be used to search for CEGP signatures in high resolution $\mathrm{K}$ band spectroscopic timeseries observations. Here, both HD 189733 (K1V-K2V) and HD 179949 (F8V) are discussed.

\section{METHOD}

The phase dependent nature of the combined star+planet spectrum is represented in Fig. 1 for a small spectral region at two orbital phases. Rather than attempting to identify molecules from their broadband effects on spectra, here a method which attempts to identify molecules through a statistical examination of the many thousand individual transitions found in a typical high resolution ( $\mathrm{R} \mathrm{25,000-50,000)}$ planetary spectrum is used. Signal enhancement via deconvolution extracts the faint planetary absorption signature from individual spectra. The planetary orbit is densely sampled via timeseries observations and the phase dependent Doppler shift of the planetary spectrum is modelled. With sufficient observations, the velocity amplitude, $K_{p}$, of the planet may be determined. Since the stellar velocity amplitude, $K_{*}$ is already known, the mass ratio $q=K_{*} / K_{p}=M_{p} / M_{*}$ may be employed (with an estimate of the stellar mass) to determine the mass of the planet. Hence, the axial inclination of the orbit may be unambiguously determined. As the planet need not be transiting, this spectroscopic method increases the sample of planets which can potentially be studied with current instrumentation.

The procedure is an adaption of studies which attempted to measure the optical albedo of various hot Jupiters via a search for the planetary reflected light spectrum [12-16]. Rather than searching for a weak copy of the Doppler shifted stellar spectrum, we seek to measure the emitted planetary infrared spectrum. In the case of reflected light studies, a copy of the reflected planetary spectrum at maximum brightness is not significantly Doppler shifted from the stellar spectrum at phases close to $\phi=0.5$ and hence the system can not be studied at maximum planet brightness. Apart from more favourable contrast ratios, the main advantage of working at infrared wavelengths is that the planetary spectrum is significantly different from the stellar spectrum, enabling those phases where the planet is expected to be at it's brightest (owing to heating of the hemisphere facing the star) to be employed in the analysis.

Several hundred spectra are typically observed at high resolution, close to orbital phase $\phi=0.5$, when the heated inner face of the CEGP is visible. A residual timeseries is created by subtracting a scaled and shifted master spectrum, generated from co-addition of all spectra, from each spectrum in turn. Because the planetary spectrum is not stationary during the observations, the master spectrum contains 

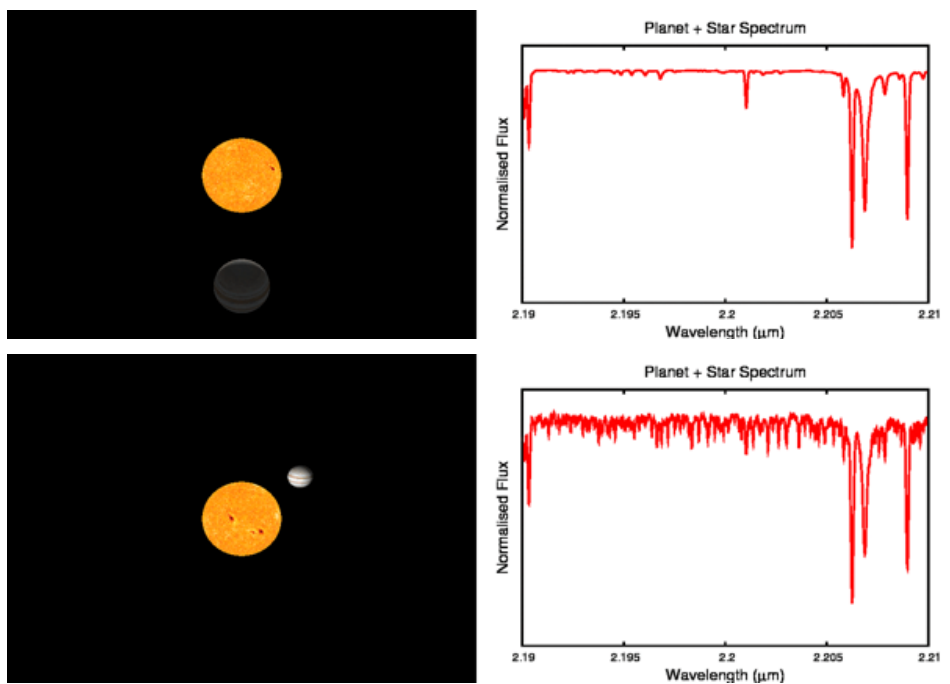

Figure 1. Representation of the location of the planet at two orbital phases, $\phi=0.0$ and 0.42 (left) and the respective combined star+planet spectra (right). The spectra are always dominated by the stationary stellar spectrum (e.g. the 3 strong lines between $2.205 \mu \mathrm{m}$ and $2.1 \mu \mathrm{m}$ ) and any telluric lines (not shown here). In the upper plot, the planet contribution to the combined spectrum is at a minimum since the nightside of the planet is seen. In the lower plot the heated dayside of the planet makes a greater contribution to the combined spectrum. The planet spectrum is Doppler shifted according to orbital phase and is shown here with an exaggerated contribution. In reality the spectral signal of any single line is hidden within the noise, and must be extracted via deconvolution.

a very faint, smeared out copy of the planetary spectrum. Accurate subtraction of the master spectrum from each spectrum in turn therefore effectively removes the relatively stationary stellar spectrum and any telluric lines. This leaves a residual that contains only a copy of the Doppler shifted planetary spectrum, which at this stage is hidden within the noise. Principal components analysis is employed to remove any remaining systematics in the residual spectra which are mainly due to imperfectly subtracted telluric features. Full details are given in [14, 17-20].

\subsection{Model spectra and deconvolution}

Model spectra for each system being investigated are generated using a self consistent cloud-free model [21]. A line list representing the molecular wavelengths and line strengths is used for deconvolution. Least squares deconvolution [14, 22, 23] is employed to make use of the hundreds to thousands of lines in model planetary atmospheres to derive a single profile with augmented $\mathrm{S} / \mathrm{N}$ ratio from each spectrum, thereby enabling planet star contrast ratios of $F_{p} / F_{*} \sim 1 / 1000-1 / 10,000$ to be probed.

\subsection{Matched filter planet search}

A Gaussian matched filter is used to model the orbit of the planet (i.e. radial velocity shift and phase dependent brightness changes) to search for an absorption/emission signature in the spectral timeseries. The model planetary signature varies in strength according to the equation

$$
\epsilon(\alpha, \lambda)=\frac{F_{p}(\alpha, \lambda)}{F_{*}(\alpha, \lambda)}=\epsilon_{0}(\lambda) g(\alpha, \lambda)
$$

where $\alpha=\sin (i) \cos (\phi), \epsilon_{0}(\lambda)$ is the maximum planet brightness, $g(\alpha, \lambda)$ is the phase function and $i$ and $\phi$ are the axial orbital inclination and planetary orbital phase respectively. Pairs of maximum contrast 

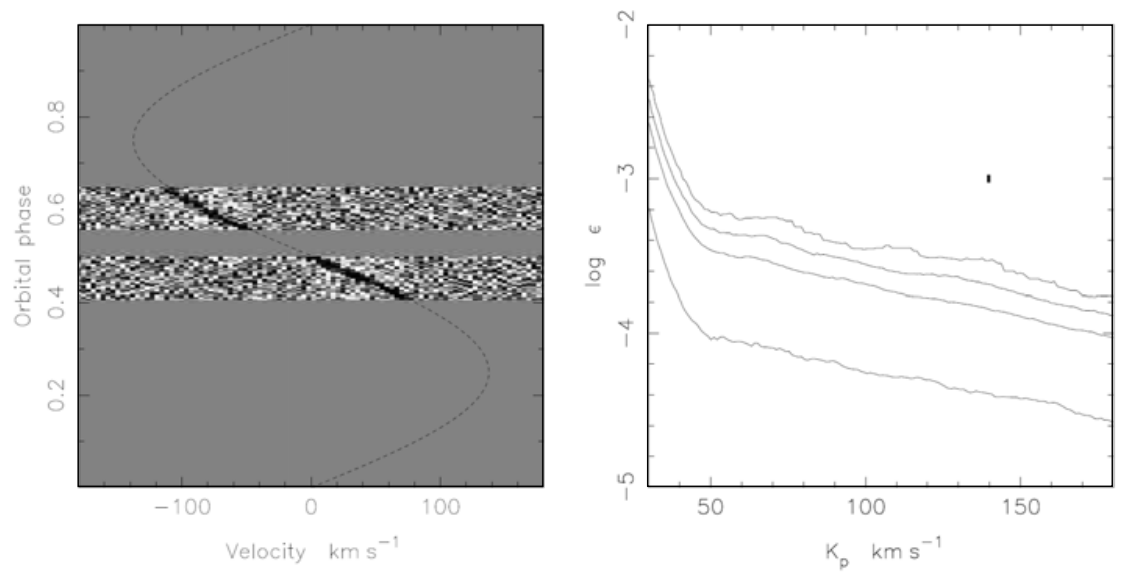

Figure 2. Right: Deconvolved timeseries for two nights of simulated observations. A model planetary signal was injected into the spectra before analysis with a maximum contrast ratio, $\epsilon_{0}=F_{p} / F_{*}=0.001$. The dashed line shows the expected phase dependent location of the Doppler shifted planetary signature which appears as a dark absorption signature in the residual spectra. Left: Pairs of maximum contrast ratio $\left(\epsilon_{0}\right)$ vs velocity amplitude $\left(K_{p}\right)$ are used in a two dimensional $\chi^{2}$ search to find the combination which yields the best improvement in $\chi^{2}$. The planet is recovered with high confidence at the simulated velocity amplitude and contrast ratio $\log _{10}\left(\epsilon_{0}\right)=-3$. Black and white levels represent the best and worst improvements in $\chi^{2}$ respectively. Upper confidence levels of 63.8, 95.4, $99 \& 99.9$ per cent (top to bottom solid lines) are plotted.

ratio $\left(\epsilon_{0}\right)$ vs velocity amplitude $\left(K_{p}\right)$ are used in a two dimensional $\chi^{2}$ search to find the combination which yields the best improvement in $\chi^{2}$. The significance of candidate enhancements in $\chi^{2}$ are assessed by a bootstrap procedure which randomises the order of the data within each night of observations [14]. This process, carried out several thousand times, scrambles any planetary signature, but enables the data to retain the ability to give false $\chi^{2}$ enhancements due to systematics which may remain in the data above the photon noise. Reliable confidence levels can thus be plotted on the 2-parameter $\chi^{2}$ landscapes of $\log _{10}\left(\epsilon_{0}\right)$ vs $\left(K_{p}\right) \chi^{2}$. Calibration of contrast ratios is achieved by injecting a "fake" model planet spectrum into the timeseries and then recovering the signal using the matched filter method. The fake planetary spectrum is injected with known $\epsilon_{0}$ and $K_{p}$ after extraction of the spectra and before any of the subsequent steps described above are carried out. In this way, the ability to correctly recover a planetary signature [18, 19] may be assessed (Fig. 2).

\section{RESULTS}

The above method is applied to two bright stars with close orbiting extrasolar giant planets. HD 179949b is expected to possess a temperature inversion [8] since it is highly irradiated by its F8V parent star. With a K0V host, the atmosphere of the well studied transiting planet, HD 189733b, is consistent with the scenario in which any high altitude absorbing species have "rained out", leaving an atmosphere dominated by absorption features (no thermal inversion).

\subsection{HD 179949b}

The presence of a close orbiting planetary system around the $K=4.9$, F8V star, HD 189733 was first reported by [24]. The respective semi-major axis and orbital period of the planet, HD 179949b, are $\mathrm{a}=0.045 \mathrm{AU}$ and $\mathrm{P}=3.09 \mathrm{~d}$. Observations were made on $2007 \mathrm{Jul} 26$ \& Aug 03 with CRIRES/VLT $(\mathrm{R} \sim 50,000)$. The mean deconvolved wavelength of the spectra is $\lambda_{\text {mean }}=2.14 \mu \mathrm{m}$. Since the planet does not transit the star, the axial inclination is unknown. A most probable inclination of $i=47.6^{\circ}$ is 
Research, Science and Technology of Brown Dwarfs and Exoplanets
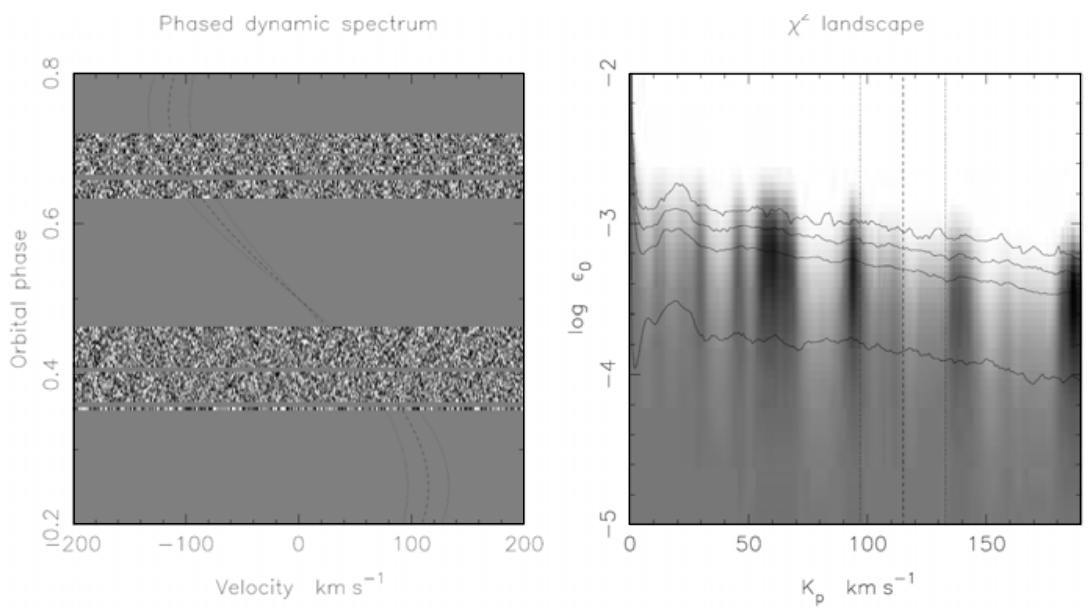

Figure 3. Phased deconvolved timeseries spectra of HD 179949b (left) and corresponding 2-dimensional $\chi^{2}$ landscape plot (right) for matched filter combinations of maximum contrast ratio, $\log _{10}\left(\epsilon_{0}\right)$ vs $K_{p}$. The dashed sinusoidal curve in the timeseries plot represents the expected motion of a planetary signal based on the most probable velocity amplitude, $\hat{K}_{p}=115 \mathrm{~km} \mathrm{~s}^{-1}$. The dotted sinusoidal curves represent the motion of a planet with $K_{p}$ which deviates by $\pm 2 \sigma$ from $\hat{K}_{p}$. The corresponding $K_{p}$ amplitudes are shown in the $\chi^{2}$ plot (right). Numerous enhancements in $\chi^{2}$ result from low level systematic residuals which are not fully removed. No high confidence planetary signal is detected at the most probable velocity amplitude, $\hat{K}_{p}$.

estimated from Monte-Carlo simulations. The timeseries and 2D $\chi^{2}$ plot are shown in Fig. 3 (see caption for details). The $\chi^{2}$ plot shows a number of enhancements in the model fit at various combinations of contrast ratio and velocity amplitude, $K_{P}$. However these features appear with low significance. The temperature inversion model used for deconvolution resembles a blackbody over the wavelength region of interest, with weak emission lines superimposed (see spectral energy distribution (SED) in Fig. 4). However, since the lines are weak, this model can not be rejected with the analysis since the 95.4 per cent upper limit of $\log _{10} \epsilon_{0}=-3.30\left(F_{p} / F_{*} \sim 1 / 2000\right)$ at the most probable velocity amplitude is higher than the model contrast ratio of $\log _{10} \epsilon_{0}=-3.46\left(F_{p} / F_{*} \sim 1 / 2900\right)$ at the mean wavelength of the observations.

\subsection{HD 189733b}

Important system parameters of HD 189733b, are: orbital semi major axis, $a=0.031 \mathrm{AU}$, orbital period, $P=2.21 \mathrm{~d}$, axial inclination, $i=85.76^{\circ}$. Observations were made on 2008 June $15 \& 22$ with NIRSPEC at Keck $2(\mathrm{R} \sim 25,000)$. These data were taken under much more favourable conditions than was available in a previous 2006 Jul 22 data set $(\mathrm{R} \sim 15,000)$ [18]. Recent Spitzer/IRAC and HST/NICMOS observations of HD 189733b [4] have enabled measurement of the dayside spectrum using low resolution spectroscopy. In order to reliably fit the spectrum, a greater than expected abundance of $\mathrm{CO}_{2}$ is required (mixing ratios of $c \sim 0.1-1 \times 10^{-6}$ ). For deconvolution, a model (matching the HST/NICMOS observations) with augmented $\mathrm{CO}_{2}$ abundance was used. The model does not include a temperature inversion and all atmospheric molecular species appear in absorption. This model does not contain some of the hot $\mathrm{CO}_{2}$ bands which have been identified by Fourier Transform Spectroscopy carried out at the Jet Propulsion Laboratory and included in the latest edition of HITRAN (see [25] and references therein). In order to achieve the required level of absorption from $\mathrm{CO}_{2}$ in the 1.9-2.2 $\mu \mathrm{m}$, region, the relative strengths of individual opacities in the output model are likely overestimated. Nevertheless, based on the NICMOS results, inclusion of such opacities would appear to give a more accurate representation of the expected opacities found in the spectrum of HD 189733b. 


\section{EPJ Web of Conferences}

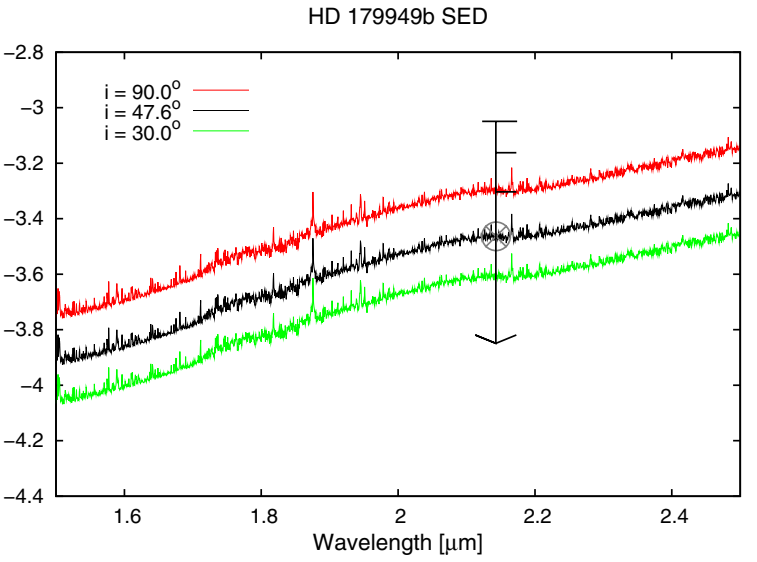

Figure 4. HD 179949b planet/star flux ratios at $2.14 \mu \mathrm{m}$. Some apparent emission features are due to stellar absorption lines and result from dividing the planet spectrum by the stellar spectrum. The grey circled crosses indicate the mean model flux over the wavelength of observations for the most probable inclination. Upper limits corresponding to the levels in Fig. 3 (99.9, 99 and 95.4 per cent) are marked by horizontal bars (from top to bottom) for our analysis using each model. The arrow head marks the 68.3 per cent $(1-\sigma)$ confidence level. The width of the top horizontal bar (99.9 percent confidence) represents the wavelength range of the CRIRES spectroscopic observations. The model at the most probable inclination $\left(47.6^{\circ}\right)$ can not be excluded since the 95.4 per cent confidence level is above the model flux ratio and residuals (Fig. 3) are present at 68.3-95.4 per cent confidences.
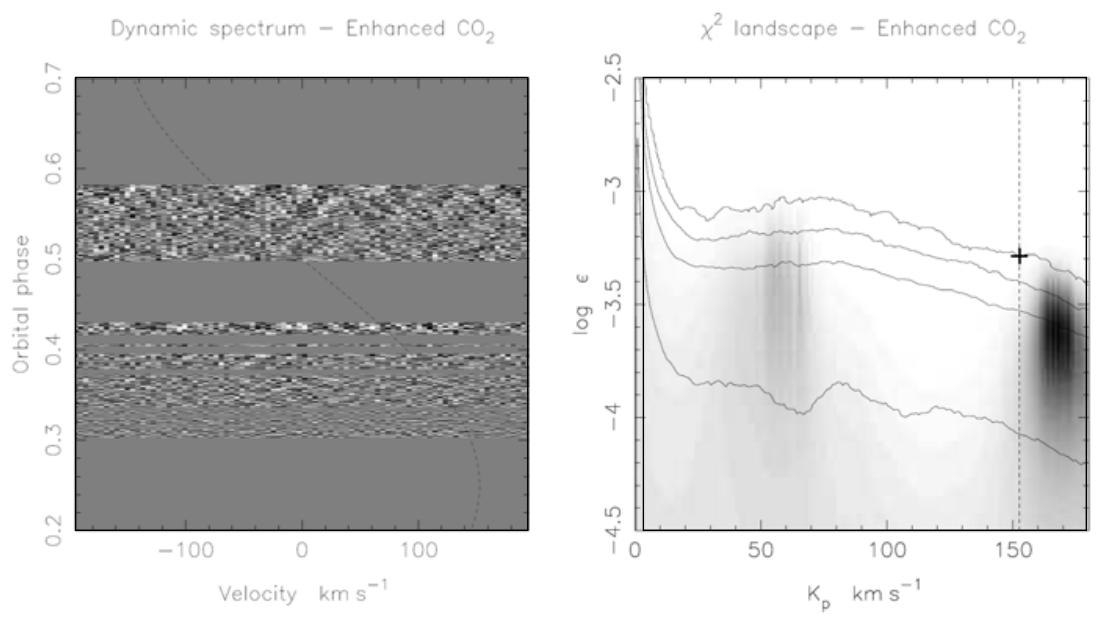

Figure 5. As for Fig. 3, plotted for HD 189733b. Since HD 189733b is a transiting planet, the orbital inclination and hence velocity amplitude, $K_{p}=152.6 \mathrm{~km} \mathrm{~s}^{-1}$, of the planet are known. The bold + symbol marks the mean value of the contrast ratio over the range of observations for the NICMOS/HST [4] observations.

Figure 5 shows the deconvolved timeseries (mean wavelength $\lambda=2.15 \mu \mathrm{m}$ ) and the $\chi^{2}$ plot of $\log \left(\epsilon_{0}\right)$ vs $K_{p}$. At $K_{p}=152.6 \mathrm{kms}^{-1}$, the expected planetary signature is not detected with 68.3 , 95.4, 99 and 99.9 per cent confidence levels of $\log _{10}\left(\epsilon_{0}\right)=-4.074,-3.529 \&-3.400 \&-3.283$ or $F_{p} / F_{*}=1 / 11600,1 / 3380,1 / 2510 \& 1 / 1920$ respectively. The reported mean HST/NICMOS 2.0$2.4 \mu \mathrm{m}$ planet/star flux ratio is $\log _{10}\left(\epsilon_{0}\right)=-3.286$ or $F_{p} / F_{*}=1 / 1930$ [4] (i.e. almost identical to the 99.9 per cent confidence level of 1/1920). Figure 6 shows the SED for HD 189733b. A fuller analysis can be found in [20]. 
HD 189733b SED

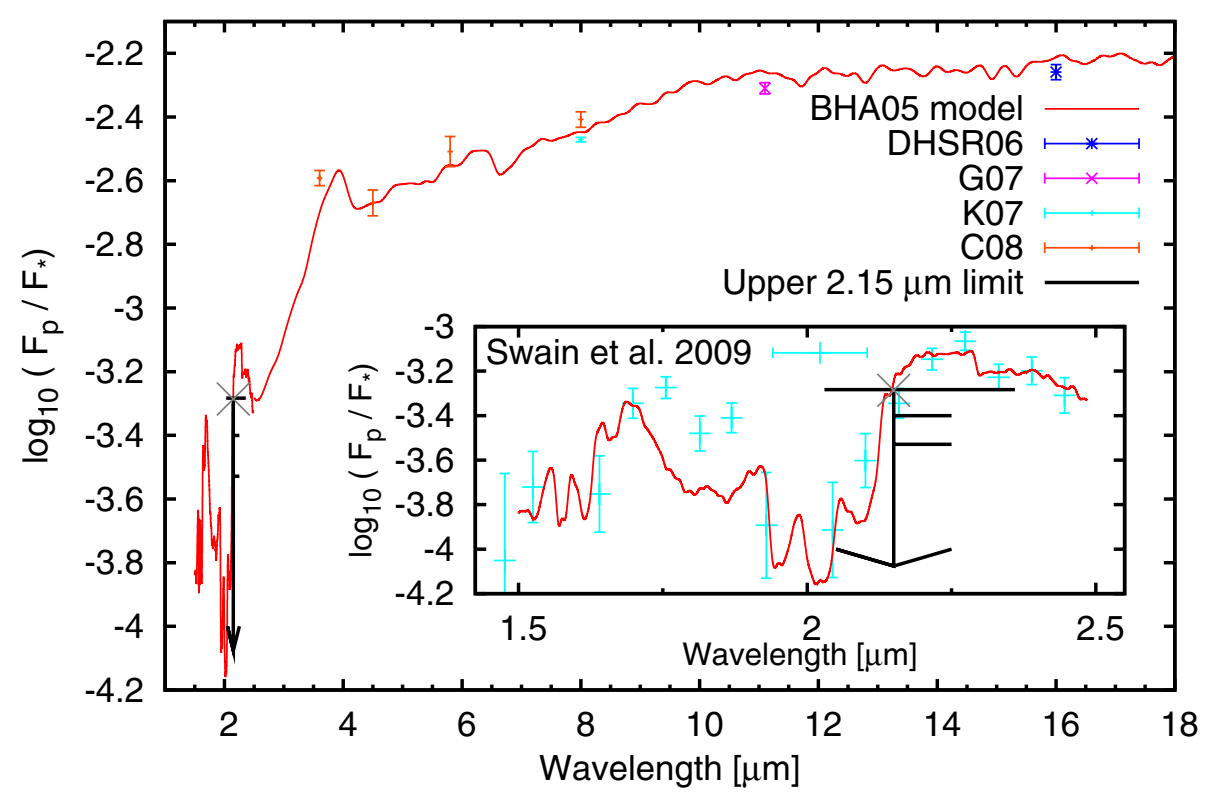

Figure 6. As for Fig. 4, but for HD 189733b, showing the good match of the model to the numerous observations. The inset shows a blow-up of the 1.5-2.5 $\mu \mathrm{m}$ region and shows the NICMOS/HST [4] spectrum. References are BHA05 [21], DHSR06 [27], G07 [28], K07 [29], C08 [30].

The question of whether the low-significance candidate signature in Fig. 5 could be due to the planet has been investigated at length in [20]. By splitting the data into two independent wavelength regions, a candidate planetary signature within $11 \mathrm{~km} \mathrm{~s}^{-1}$ of the known velocity amplitude and between 94.8 and 98.8 significance was found. Tests reveal that signatures with low significance such as these are effected by any residuals which remain in the data and which can also mimic a planetary signature through chance alignment. The source of residuals is most likely dominated by imperfect template subtraction owing largely to variations in the spectrum due to changing telluric strengths. While the detected HD $189733 \mathrm{~b}$ candidate signature may indeed by due to the planet, it is clear that greater confidence is required to unambiguously detect a signal.

\section{DISCUSSION}

The $\mathrm{K}$ band observations are not sensitive enough to detect the atmospheric signature of HD 179949b at the most probable velocity amplitude and inclination. Detection of the molecular emission features arising from an atmosphere with a temperature inversion is more difficult than for a cooler atmosphere dominated by stronger absorption signatures. The study of HD $189733 \mathrm{~b}$ however has achieved a sensitivity which should be sufficient to detect $\mathrm{H}_{2} \mathrm{O}, \mathrm{CO}$ and $\mathrm{CO}_{2}$ absorption features, at high resolution, with reasonable significance ( 99.9 per cent). Given the good agreement between photometric space based observations and the models, the lack of detection of HD 189733b may be explained by a mismatch at high resolution between the model spectrum used for deconvolution and the true empirical spectrum. The effects of observational and model uncertainties have been investigated to determine to what extent a planetary signature may appear weaker than expected following the deconvolution procedure. Opacity strength uncertainties were investigated by modifying the model planet temperaturepressure profiles in an ad hoc way. Similarly, use of model line lists of water [26] were used to 
determine the non-negligible effects of wavelength uncertainties. The effects of model opacity strength uncertainties, wavelength uncertainties, temperature mismatch, phase function mismatch and velocity field/broadening uncertainties contribute sensitivity uncertainties of 12.5, 14.5, 20.5, 4.7 and 9.6 percent respectively. Combining these effects in quadrature yields a total uncertainty in the significance of the result of 30 per cent. Further, if we take a semi-empirical 55 per cent uncertainty as an upper limit (derived by deconvolving an L dwarf spectrum with similar temperature to HD 189733b) to the line depth, wavelength and temperature mismatches, the corresponding uncertainty is 56 per cent. Assuming that the L spectrum can provide a close match to that of HD $189733 \mathrm{~b}$, the semi-empirical result already shows that the model uncertainties may be significantly underestimated. Hence the 99.9 percent confidence with which a signal at the known $K_{p}$ can be rejected could in fact be modified to a level with reduced significance, taking a candidate signal to contrast ratios that are plagued by systematic features.

While the current generation of models can adequately fit broadband photometric and low resolution spectroscopic observations, it is clear that moving to higher resolution requires further model refinement. With the uncertainties investigated above, the presence of HD 189733b can not be ruled out using the technique described here, especially if further model uncertainties remain unaccounted for. Only further observations, which would bring about an increase in sensitivity, or more accurate model atmospheres, could increase the chances of detecting close orbiting extrasolar giant planets.

\section{References}

[1] T. Barman, ApJ 661, L191 (2007), arXiv: 0704.1114

[2] G. Tinetti, A. Vidal-Madjar, M.C. Liang, J.P. Beaulieu, Y. Yung, S. Carey, R.J. Barber, J. Tennyson, I. Ribas, N. Allard et al., Nature 448, 169 (2007), arXiv : 0707 . 3064

[3] M.R. Swain, G. Vasisht, G. Tinetti, Nature 452, 329 (2008)

[4] M.R. Swain, G. Vasisht, G. Tinetti, J. Bouwman, P. Chen, Y. Yung, D. Deming, P. Deroo, ApJ 690, L114 (2009)

[5] M.R. Swain, G. Tinetti, G. Vasisht, P. Deroo, C. Griffith, J. Bouwman, P. Chen, Y. Yung, A. Burrows, L.R. Brown et al., ApJ 704, 1616 (2009), arXiv: 0908.4010

[6] A. Burrows, J. Budaj, I. Hubeny, ApJ 678, 1436 (2008), arXiv: 0709.4080

[7] I. Hubeny, A. Burrows, D. Sudarsky, ApJ 594, 1011 (2003), arXiv: astro-ph/0305349

[8] J.J. Fortney, K. Lodders, M.S. Marley, R.S. Freedman, ApJ 678, 1419 (2008), arXiv : 0710.2558

[9] A. Burrows, I. Hubeny, D. Sudarsky, ApJ 625, L135 (2005), arXiv : astro-ph/0503522

[10] A. Burrows, I. Hubeny, J. Budaj, H.A. Knutson, D. Charbonneau, ApJ 668, L171 (2007), arXiv:0709.3980

[11] H.A. Knutson, D. Charbonneau, L.E. Allen, A. Burrows, S.T. Megeath, ApJ 673, 526 (2008), arXiv: 0709.3984

[12] A. Collier Cameron, K. Horne, A. Penny, D. James, Nature 402, 751 (1999), astro-ph/9911314

[13] D. Charbonneau, R.W. Noyes, S.G. Korzennik, P. Nisenson, S. Jha, S.S. Vogt, R.I. Kibrick, ApJ 522, L145 (1999), astro-ph/9907195

[14] A. Collier Cameron, K. Horne, A. Penny, C. Leigh, MNRAS 330, 187 (2002), astro-ph/0110577

[15] C. Leigh, A.C. Cameron, K. Horne, A. Penny, D. James, MNRAS 344, 1271 (2003a), astro-ph/0308413

[16] C. Leigh, A. Collier Cameron, S. Udry, J.F. Donati, K. Horne, D. James, A. Penny, MNRAS 346, L16 (2003b), astro-ph/0310489

[17] J.R. Barnes, C.J. Leigh, H.R.A. Jones, T.S. Barman, D.J. Pinfield, A. Collier Cameron, J.S. Jenkins, MNRAS 379, 1097 (2007a), arXiv: 0705.0272

[18] J.R. Barnes, T.S. Barman, L. Prato, D. Segransan, H.R.A. Jones, C.J. Leigh, A. Collier Cameron, D.J. Pinfield, MNRAS 382, 473 (2007b), arXiv: 0708.4300 
[19] J.R. Barnes, T.S. Barman, H.R.A. Jones, C.J. Leigh, A.C. Cameron, R.J. Barber, D.J. Pinfield, MNRAS 390, 1258 (2008), 0806.0298

[20] J.R. Barnes, T.S. Barman, H.R.A. Jones, R.J. Barber, B.M.S. Hansen, L. Prato, E.L. Rice, C.J. Leigh, A. Collier Cameron, D.J. Pinfield, ArXiv e-prints (2009), 0909 . 2510

[21] T.S. Barman, P.H. Hauschildt, F. Allard, ApJ 632, 1132 (2005), astro-ph/0507136

[22] J.F. Donati, M. Semel, B. Carter, D.E. Rees, A. Collier Cameron, MNRAS 291, 658 (1997)

[23] J.R. Barnes, A. Collier Cameron, Y.C. Unruh, J.F. Donati, G.A.J. Hussain, MNRAS 299, 904 (1998)

[24] C.G. Tinney, R.P. Butler, G.W. Marcy, H.R.A. Jones, A.J. Penny, S.S. Vogt, K. Apps, G.W. Henry, ApJ 551, 507 (2001), arXiv: astro-ph/0012204

[25] L.S. Rothman, I.E. Gordon, A. Barbe, +40 co-authors, Journal of Quantitative Spectroscopy and Radiative Transfer 110, 533 (2009)

[26] R.J. Barber, J. Tennyson, G.J. Harris, R.N. Tolchenov, MNRAS 368, 1087 (2006), arXiv:astro-ph/0601236

[27] D. Deming, J. Harrington, S. Seager, L.J. Richardson, ApJ 644, 560 (2006), astro-ph/0602443

[28] C.J. Grillmair, D. Charbonneau, A. Burrows, L. Armus, J. Stauffer, V. Meadows, J. Van Cleve, D. Levine, ApJ 658, L115 (2007), arXiv: astro-ph/0702494

[29] H.A. Knutson, D. Charbonneau, L.E. Allen, J.J. Fortney, E. Agol, N.B. Cowan, A.P. Showman, C.S. Cooper, S.T. Megeath, Nature 447, 183 (2007), 0705.0993

[30] D. Charbonneau, H.A. Knutson, T. Barman, L.E. Allen, M. Mayor, S.T. Megeath, D. Queloz, S. Udry, ApJ 686, 1341 (2008), 0802.0845 